\title{
Pseudoaneurisma roto de artéria ileocólica: relato de caso
}

\author{
Ruptured ileocolic artery pseudoaneurysm: case report \\ Carolina Vasconcellos Sant'Anna', Felipe Garcia Kuhl', Alane Miranda Leite', Selma Regina de Oliveira Raymundo' (D), \\ André Rodrigo Miquelin', Vitória Acar', Vitor Brumato Fachini', Matheus Rafael Canuti'
}

\begin{abstract}
Resumo
Os aneurismas de artérias viscerais (AAVs) e pseudoaneurismas de artérias viscerais (PAAVs) são condições raras e potencialmente letais quando rotos. Em geral, são encontrados incidentalmente na tomografia computadorizada de pacientes assintomáticos. Embora a cirurgia aberta convencional seja considerada atualmente o tratamento padrão ouro, a abordagem endovascular vem ganhando relevo por ser considerada um procedimento minimamente invasivo e com riscos cirúrgicos menores. Destaca-se, nessa abordagem, o uso de embolização por molas (coils) em AAVs e PAAVs saculares e implante de stent modulador de fluxo como alternativa de tratamento para aneurismas fusiformes. Apresentamos o caso de uma paciente de 51 anos com queixa de dor abdominal aguda, taquicardia e hipotensão, com evidência de sangramento abdominal em angiotomografia e diagnóstico de pseudoaneurisma de artéria ileocólica (AIC) roto. Ela foi submetida a tratamento endovascular precoce de embolização da AIC com sucesso, e houve melhora do quadro clínico.
\end{abstract}

Palavras-chave: procedimento endovascular; falso aneurisma; artéria mesentérica superior.

\begin{abstract}
Visceral artery aneurysms (VAAs) and visceral artery pseudoaneurysms (VAPAs) are rare conditions and are potentially lethal when they rupture. They are usually found as incidental findings on computed tomography (CT) scans of asymptomatic patients. Although conventional open surgery is currently considered the gold standard treatment, the endovascular approach has gained prominence as a minimally invasive procedure with lower surgical risk. In this approach, use of coil embolization in saccular VAAs and VAPAs and implantation of flow-modulating stents constitute alternative treatments for fusiform aneurysms. We present the case of a 51-year-old female patient complaining of acute abdominal pain, tachycardia, and hypotension, with evidence of abdominal bleeding on CT angiography, who was diagnosed with a ruptured ileocolic artery (ICA) pseudoaneurysm. She underwent early endovascular treatment for ICA embolization, which was successful and achieved clinical improvement.
\end{abstract}

Keywords: endovascular procedure; pseudoaneurysm; superior mesenteric artery.

Como citar: Sant'Anna CV, Kuhl FG, Leite AM, et al. Pseudoaneurisma roto de artéria ileocólica: relato de caso. J Vasc Bras. 2021;20:e20210163. https://doi.org/10.1590/1677-5449.210163

${ }^{1}$ Faculdade de Medicina de São José do Rio Preto - FAMERP, São José do Rio Preto, SP, Brasil.

Fonte de financiamento: Nenhuma.

Conflito de interesse: Os autores declararam não haver conflitos de interesse que precisam ser informados.

Submetido em: Agosto 23, 2021. Aceito em: Novembro 10, 2021.

O estudo foi realizado no Hospital de Base pela Instituição Faculdade de Medicina de São José do Rio Preto (FAMERP), São José do Rio Preto, SP, Brasil.

Copyright(C) 2021 Os autores. Este é um artigo publicado em acesso aberto (Open Access) sob a licença Creative Commons Attribution, que permite uso, distribuição e reprodução em qualquer meio, sem restrições desde que o trabalho original seja corretamente citado. 


\section{INTRODUÇÃO}

Os aneurismas de artérias viscerais (AAVs) e pseudoaneurismas de artérias viscerais (PAAVs) são raros, com incidência entre 0,01 e $0,2 \%{ }^{1}$. Com o avanço de técnicas em exames de imagem como a angiotomografia computadorizada (angioTC) de abdômen e o ultrassom Doppler (USD) ${ }^{2,3}$ para detecção de doenças intra-abdominais, juntamente com maior manipulação das vias biliares, a taxa de prevalência e o achado incidental dos aneurismas têm aumentado. Os AAVs são causados principalmente por hipertensão, aterosclerose, displasia e doenças do colágeno, enquanto os PAAVs estão associados a traumas, lesões iatrogênicas, inflamação ou infecções ${ }^{4}$. A artéria esplênica é a mais comumente afetada por aneurismas e pseudoaneurismas, seguida pela artéria hepática, tronco celíaco e artéria mesentérica inferior ${ }^{5}$.

Os PAAVs ocorrem mais frequentemente nas artérias gastroduodenal e mesentérica superior (AMS) se comparados a aneurismas verdadeiros, e os pseudoaneurismas rotos dessa artéria estão associados à taxa de mortalidade de $37 \%{ }^{6}$.

Os aneurismas e pseudoaneurismas das artérias ileocólicas (AIC) são incomuns e geralmente assintomáticos, mas, quando sintomáticos, estão associados a sinais de rotura, tais como dor abdominal, sangramento intestinal baixo e hemorragia intraabdominal ou sinais de choque hipovolêmico ${ }^{7}$. A cirurgia convencional é considerada padrão ouro no tratamento, porém o tratamento endovascular (TE) vem se destacando, principalmente na abordagem de pacientes com alto risco cirúrgico ${ }^{8,9}$.

Apresentamos o caso de uma paciente diagnosticada com PAAV de AIC roto submetida a TE com sucesso. Ressaltamos a importância do conhecimento dessa condição, que, embora rara e potencialmente fatal, pode apresentar um desfecho favorável se diagnosticada e tratada precocemente.

O estudo foi aprovado pelo Comitê de Ética e Pesquisa da nossa instituição (CAAE: 50239621.8.0000.5415, número do parecer consubstanciado: 4.919.534).

\section{Descrição do caso}

Paciente do sexo feminino de 51 anos admitida em pronto atendimento com dor abdominal de forte intensidade em região superior há 2 dias e piora com o passar das horas, sem melhoria com uso de analgésicos. Na admissão, apresentava regular estado geral, palidez cutânea-mucosa, pele fria, pressão arterial de 125 x $59 \mathrm{mmHg}$, frequência cardíaca de $116 \mathrm{bpm}$, frequência respiratória de $17 \mathrm{ipm}$ e temperatura corporal de $36,1^{\circ}$. No exame físico, o abdômen mostrava-se plano, com dor leve à palpação profunda na região superior, ausência de massa e/ ou dor à descompressão. Foi solicitada angioTC abdominal, evidenciando-se volumoso hematoma intraperitoneal no mesentério na região do mesogastro de aproximadamente $800 \mathrm{~cm}^{3}$ e presença de líquido livre na cavidade abdominal em volume moderado. A AMS encontrava-se pérvia, e observou-se uma dilatação fusiforme em AIC com irregularidade de contornos e extravasamento de contraste ao longo do seu trajeto à direita (Figura 1A e 1B). A paciente negava doenças prévias e uso de medicamentos contínuos. Referiu ter sido submetida à colecistectomia videolaparoscópica há vários anos.

Posteriormente, a paciente foi encaminhada ao setor de hemodinâmica, sendo realizada arteriografia por técnica de Seldinger com passagem de válvula hemostática 5Fr Performa ${ }^{\circledR}$ (Merit Medical Systems, Inc., Utah, EUA) e posicionamento de cateter Cobra Performa ${ }^{\circledR}$ (Merit Medical Systems, Inc., Utah, EUA), com injeção de contraste que identificou dilatação e extravasamento de contraste em AIC (Figura 2A e 2B). Foi também realizado cateterismo seletivo da AIC com microcateter Progreat (Terumo Corporation, Tóquio, Japão), e optou-se por embolização dessa artéria com liberação de micromolas Axium Prime ${ }^{\mathrm{TM}}$ (Medtronic, Dublin, Irlanda) de 2,5 x $6 \mathrm{~cm}$ em região final da dilatação e de micromolas Axium $^{\mathrm{TM}}$ (Medtronic, Dublin, Irlanda) de 2,5 x $8 \mathrm{~cm}$ com selamento de outflow, seguido por liberação de molas de embolização Nester ${ }^{\circledR}$ (Cook Medical Inc., Indiana, EUA) em área de extravasamento de contraste ( 5 molas 14 x $20 \mathrm{~mm}$ e 2 molas 14 x $18 \mathrm{~mm}$ ), com selamento de inflow (Figura 3). Após o procedimento, a paciente apresentou melhora da dor e estabilidade hemodinâmica e recebeu alta hospitalar no $3^{\circ}$ dia do pós-operatório (PO). No retorno de 3 meses, a paciente apresentava-se assintomática, e foi realizada angioTC controle, não sendo observados extravasamentos ou hematoma retroperitoneal (Figura 4A e 4B).

\section{Discussão}

Os AAVs e PAAVs são manifestações raras, com incidência entre 0,1 e $2 \%^{1}$, mas, apesar da baixa incidência, apresentam taxa de ruptura alta (25\%) com morbimortalidade significante $(70 \%)^{5,10}$. São causados por uma variedade de etiologias, e o diagnóstico inicial inclui USD e tomografia computadorizada (TC) com sensibilidade de 50\% e $67 \%$, respectivamente. A angiografia é o padrão ouro, pois permite a localização mais específica da lesão e confirmação do fluxo colateral, podendo ser usada para tratamento ${ }^{11}$. Os PAAVs de AMS são mais frequentemente causados por inflamação devido à pancreatite ou outras etiologias, além de poderem 


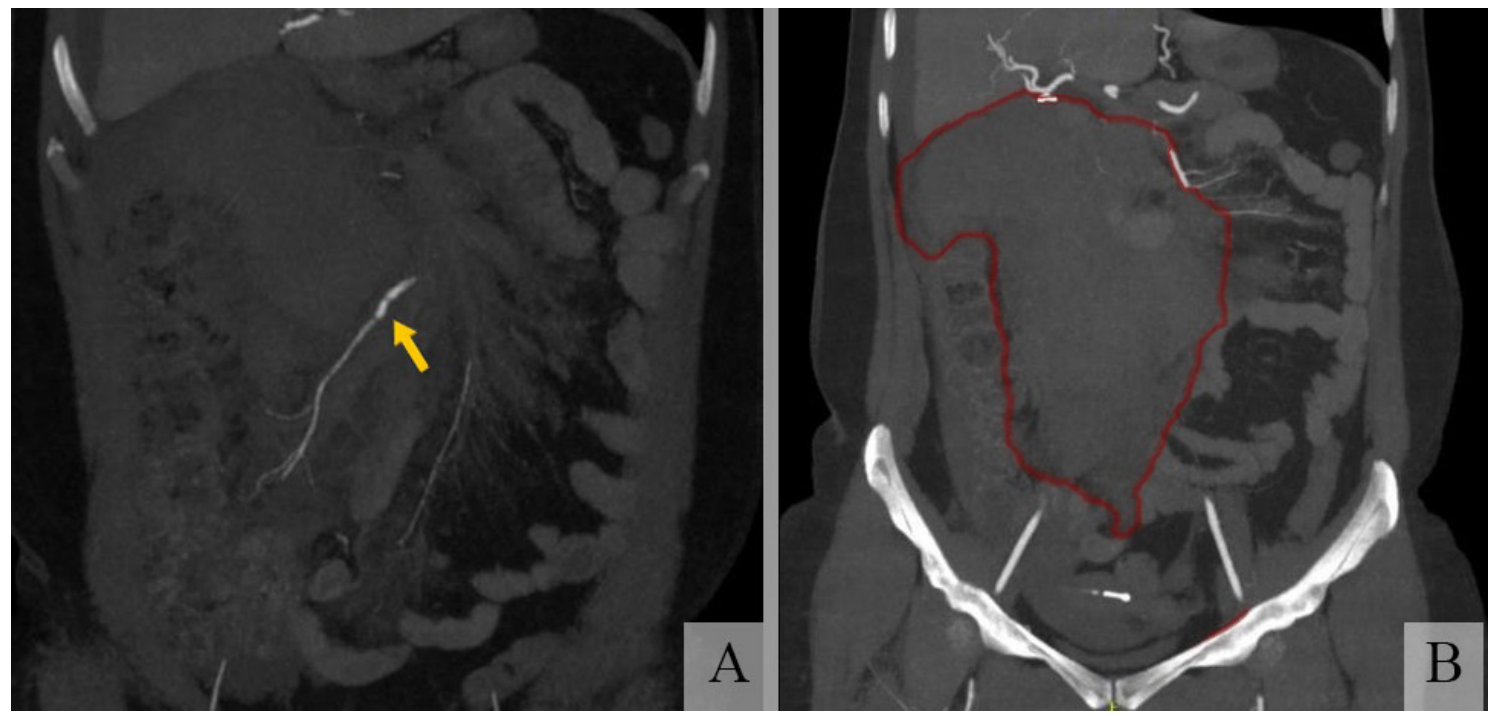

Figura 1. Angiotomografia de abdômen evidenciando: (A) artéria mesentérica superior apresentando dilatação fusiforme em artéria ileocólica com irregularidade de contornos e extravasamento de contraste; (B) volumoso hematoma intraperitoneal no mesentério e presença de líquido livre na cavidade abdominal.

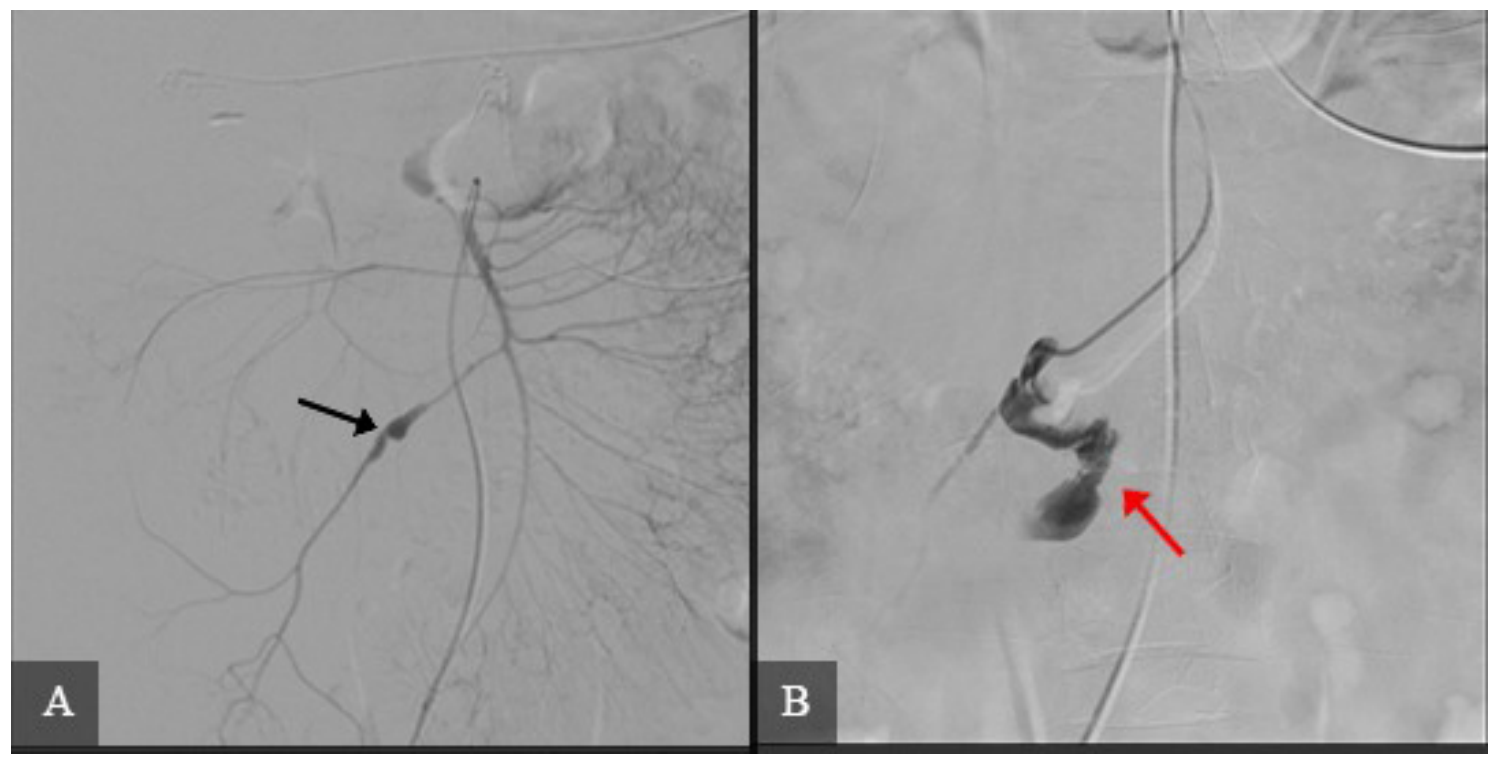

Figura 2. Angiografia de artérias abdominais mostrando: (A) pseudoaneurisma fusiforme em porção média da artéria ileocólica (seta preta); (B) extravasamento de contraste indicando rotura (seta vermelha).

ser causados por traumas de origem iatrogênica ${ }^{6}$ desde cirurgia ou angiografia até acidentes ou traumas penetrantes ${ }^{11}$. A maior manipulação do trato biliar com técnicas percutâneas endoscópicas e implantes de cateter para quimioembolização intravascular resultaram em aumento da incidência de degeneração pseudoaneurismal de vasos viscerais, além de trauma arterial após tratamento laparoscópico de doenças intra e retroperitoneais ${ }^{12}$.
Com a disseminação das técnicas de imagem, como angioTC e angiorressonância magnética, houve um aumento no diagnóstico de AAVs e PAAVs e, muitas vezes, são achados incidentais em exames realizados para outras doenças abdominais ${ }^{10}$. A apresentação clínica dessas lesões é frequentemente vaga, e não há sinais claros alertando sobre a iminência de ruptura. Recomendam-se diagnóstico e manejo rápido em qualquer suspeita de PAAV em função de sua alta taxa 
de ruptura e hemorragia ${ }^{11}$. Pitton et al. ${ }^{13}$ constataram que PAAVs são mais propensos à ruptura que aneurismas verdadeiros ( $76,3 \%$ vs. $3,1 \%$, respectivamente).

Houve, então, a necessidade de classificação para se decidir qual procedimento realizar: cirúrgico (aberto ou embolização por cateter) ou seguimento

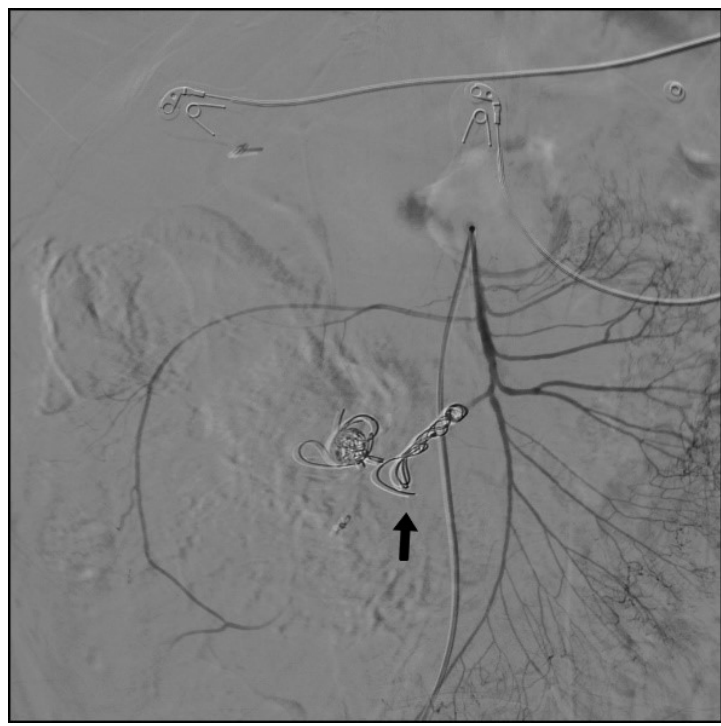

Figura 3. Angiografia de controle após embolização da artéria ileocólica com micromolas evidenciando selamento do outflow e inflow e ausência de extravasamento (seta preta). conservador ${ }^{14}$. A técnica aberta convencional que inclui ressecção com revascularização, ligadura ou ressecção final do órgão (em geral, esplenectomia) ainda permanece como padrão ouro de tratamento ${ }^{8,15}$. No entanto, pode, muitas vezes, interromper o fluxo sanguíneo da artéria principal e resultar em um quadro de isquemia intestinal em diferentes escalas ${ }^{14}$.

Tulsyan et al. ${ }^{12}$ demonstraram que a cirurgia convencional em locais de difícil acesso, com dificuldades técnicas e comorbidades associadas que não possibilitam a reconstrução vascular e em pacientes admitidos em situações críticas (sangramento agudo e rotura), pode potencializar a morbimortalidade. Eles evidenciaram taxa de sucesso de $96 \%$ na embolização endovascular com molas ${ }^{12}$.

Diante de algumas limitações na cirurgia aberta, o procedimento endovascular, por ser minimamente invasivo, tornou-se uma alternativa para pacientes que apresentavam maiores riscos cirúrgicos ${ }^{8,9,16}$. No caso de pacientes hemodinamicamente estáveis, o TE permanece a primeira opção para sangramento arterial conhecido e é considerado seguro e efetivo para tratar PAAVs. Uma vantagem para esse tipo de abordagem é permitir a individualização do tratamento para afecções diferentes, o que torna o método mais preciso e reduz as morbidades no PO. As complicações incluem ruptura do próprio saco, isquemia distal do saco pseudoaneurismático e possível fluxo arterial
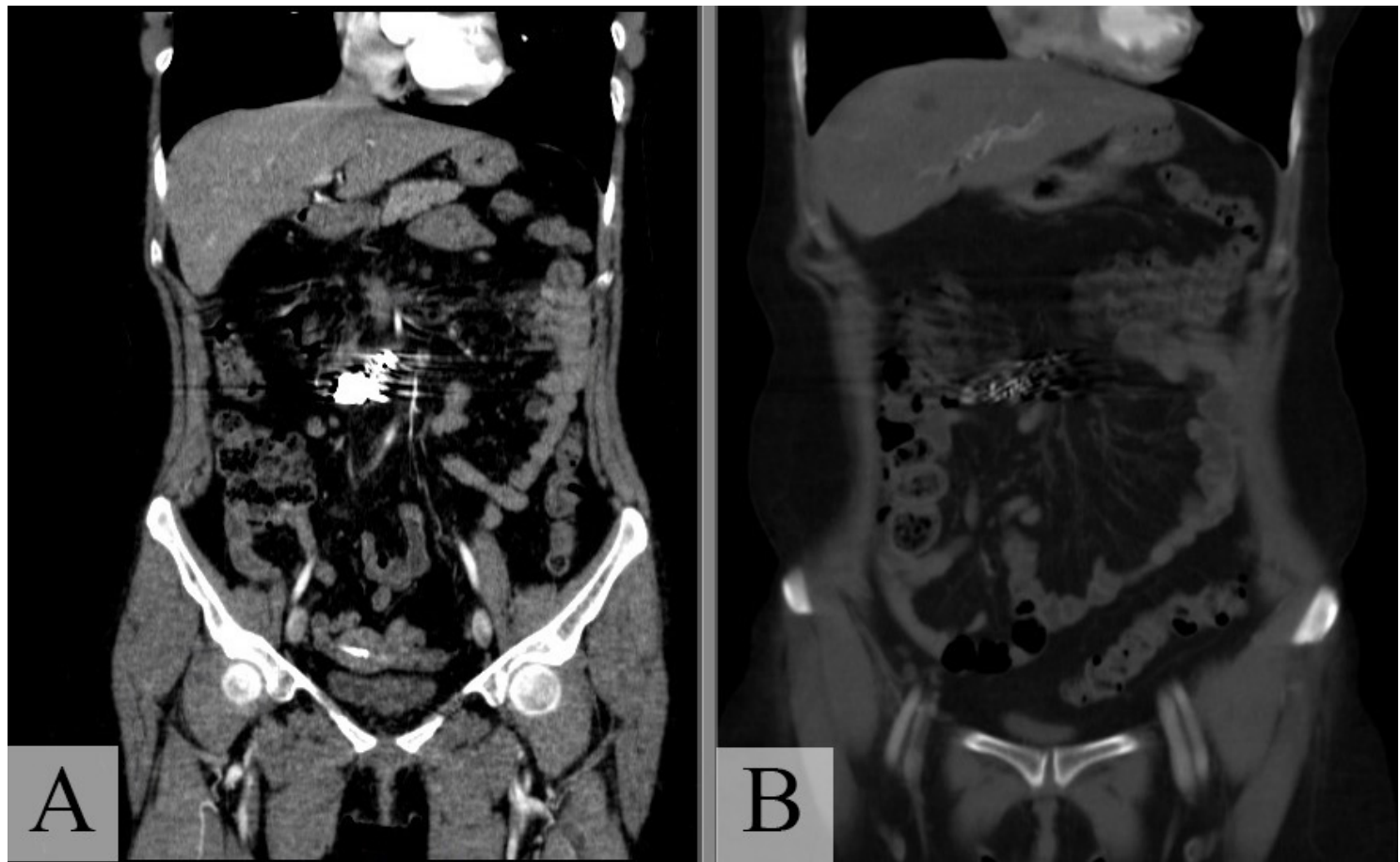

Figura 4. Angiotomografia controle após 3 meses do procedimento: (A) evidenciando as micromolas localizadas na artéria ileocólica e ausência de extravasamento; (B) imagem total do abdômen sem hematoma retroperitoneal. 
para o pseudoaneurisma após demorada reconstrução do fluxo (síndrome pós-embolização) ${ }^{11}$.

Uma variedade de materiais que promovem a oclusão da artéria está disponível, como molas, cola, plugues destacáveis, trombina, gelfoam, balões destacáveis e embolização de copolímero ${ }^{11,17,18}$. Uma das principais técnicas de TE é a embolização por molas em aneurismas e PAAVs saculares sem interrupção do fluxo da artéria principal ${ }^{14}$. O implante de stent modulador de fluxo também é uma alternativa de tratamento para aneurismas fusiformes com diâmetros largos e pequena tortuosidade ${ }^{14}$. Quando o anel aneurismático é estreito, uma opção é a embolização por molas guiadas por cateter, sendo destacáveis ou não. Entre os tipos de molas, ressaltam-se as mais recentes, que são destacáveis e constituídas de platina, um material mais maleável com forma helicoidal ${ }^{18}$.

No caso descrito aqui, depois da realização de angiografia e cateterização seletiva da AIC, foi feita embolização dessa artéria com micromolas para selamento do saco aneurismático e, na sequência, liberação de molas de embolização Nester ${ }^{\circledR}$ (Cook Medical Inc., Indiana, EUA) de platina e fibras de dácron de liberação controlada na área de extravasamento de contraste. A angiografia de controle evidenciou ausência de extravasamento de contraste e preservação de demais artérias da arcada intestinal e da AMS. A escolha pelo uso das molas descritas ocorreu em razão da compatibilidade que elas proporcionam dentro do saco pseudoaneurismático, evitando, assim, uma possível recanalização do $\mathrm{PAAV}^{18}$. O tratamento endovascular nesse caso se mostrou satisfatório e seguro, com a resolução dos sintomas da paciente e alta precoce no $3^{\circ}$ do PO.

\section{CONCLUSÃO}

O caso descrito revela a importância do reconhecimento e abordagem precoce de PAAVs com TE e utilização de molas, com redução da morbimortalidade de pacientes com abdômen agudo hemorrágico de artérias viscerais. Embora seja uma condição rara, situações semelhantes à apresentada acarretam alta letalidade em função da rotura, sendo relevante a disseminação da técnica e aprofundamento de estudos acerca do tema. Acompanhamentos a longo prazo com maior número de pacientes serão necessários para elucidar o papel final da terapia endoluminal no tratamento dos aneurismas e pseudoaneurismas viscerais.

\section{REFERÊNCIAS}

1. Chiesa R, Astore D, Guzzo G, et al. Visceral artery aneurysms. Ann Vasc Surg. 2005;19(1):42-8. http://dx.doi.org/10.1007/s10016-0040150-2. PMid:15714366.
2. Moraes D Fo, Trevisan FB, Silvestre JMS, et al. Vascular ultrasonography for follow-up of endovascular repair of abdominal aorta aneurysms. J Vasc Bras. 2014;13(3):168-74. http://dx.doi.org/10.1590/jvb.2014.019.

3. Azevedo FC, Zerati AE, Blasbalg R, Wolosker N, Puech-Leão P. Comparison of ultrasonography, computed tomography, and magnetic resonance imaging with intraoperative measurements in the evaluation of abdominal aortic aneurysms. Clinics (São Paulo). 2005;60(1):21-8. http://dx.doi.org/10.1590/S180759322005000100006. PMid:15838577.

4. Sueyoshi E, Sakamoto I, Nakashima K, Minami K, Hayashi K. Visceral and peripheral arterial pseudoaneurysms. AJR Am J Roentgenol. 2005;185(3):741-9. http://dx.doi.org/10.2214/ajr.185.3.01850741. PMid:16120928.

5. Pulli R, Dorigo W, Troisi N, Pratesi G, Innocenti AA, Pratesi C. Surgical treatment of visceral artery aneurysms: a 25-year experience. J Vasc Surg. 2008;48(2):334-42. http://dx.doi.org/10.1016/j.jvs.2008.03.043. PMid: 18644480

6. Jesinger RA, Thoreson AA, Lamba R. Abdominal and pelvic aneurysms and pseudoaneurysms: imaging review with clinical, radiologic, and treatment correlation. Radiographics. 2013;33(3):E71-96. http://dx.doi.org/10.1148/rg.333115036. PMid:23674782.

7. Roche-Nagle G, O'Donnell D, O'Hanrahan T. Visceral artery aneurysms: a symptomatic aneurysm of the ileocolic artery. Vascular. 2007;15(3):162-6. http://dx.doi.org/10.2310/6670.2007.00020. PMid:17573023.

8. De Rosa A, Gomez D, Pollock JG, et al. The radiological management of pseudoaneurysms complicating pancreatitis. JOP. 2012;13(6):6606. PMid:23183395.

9. Taboada CR, Alonso JG, Cortés RP, Pelayo LV, Hernández PV, Sánchez FS. Dual endovascular repair (coils and stent) of a true aneurysm of the gastroduodenal artery. J Vasc Bras. 2020;19:e20190123. http://dx.doi.org/10.1590/1677-5449.190123. PMid:34178066.

10. Shawky MS, Tan J, French R. Gastroduodenal Artery aneurysm: a case report and concise review of literature. Ann Vasc Dis. 2015;8(4):331-3. http://dx.doi.org/10.3400/avd.cr.15-00086. PMid:26730262.

11. Saran M, Biswas S. A rare case of jejunal pseudoaneurysm presenting as acute small bowel obstruction after blunt trauma: discussion, management dilemmas, and a review of relevant literature. Cureus. 2019;11(9):e5655. http://dx.doi.org/10.7759/ cureus.5655. PMid:31700756.

12. Tulsyan N, Kashyap VS, Greenberg RK, et al. The endovascular management of visceral artery aneurysms and pseudoaneurysms. J Vasc Surg. 2007;45(2):276-83, discussion 283. http://dx.doi. org/10.1016/j.jvs.2006.10.049. PMid:17264002.

13. Pitton MB, Dappa E, Jungmann F, et al. Visceral artery aneurysms: Incidence, management, and outcome analysis in a tertiary care center over one decade. Eur Radiol. 2015;25(7):2004-14. http:// dx.doi.org/10.1007/s00330-015-3599-1. PMid:25693662.

14. Ikeda O, Tamura Y, Nakasone $Y$, Iryou Y, Yamashita Y. Nonoperative management of unruptured visceral artery aneurysms: treatment by transcatheter coil embolization. J Vasc Surg. 2008;47(6):1212-9. http://dx.doi.org/10.1016/j.jvs.2008.01.032. PMid:18440188.

15. Santos FS, Sousa KMDS, de Castro TAC, et al. Endovascular treatment of pseudoaneurysms secondary to chronic pancreatitis: reports of two cases. J Vasc Bras. 2018;17(1):71-5. http://dx.doi. org/10.1590/1677-5449.012517. PMid:29930685.

16. Cely C, Stollman NH. Lupus abdominal crisis owing to rupture of an ileocolic aneurysm with successful angiographic treatment. J Clin Gastroenterol. 2001;32(4):347-50. http://dx.doi.org/10.1097/00004836200104000-00015. PMid:11276282. 
17. Gabelmann A, Görich J, Merkle EM. Endovascular treatment of visceral artery aneurysms. J Endovasc Ther. 2002;9(1):38-47. http:// dx.doi.org/10.1177/152660280200900108. PMid:11958324.

18. Saad NE, Saad WE, Davies MG, Waldman DL, Fultz PJ, Rubens DJ. Pseudoaneurysms and the role of minimally invasive techniques in their management. Radiographics. 2005;25(Suppl 1):S173-89. http://dx.doi.org/10.1148/rg.25si055503. PMid:16227490.
Correspondência Carolina Vasconcellos Sant'Anna Rua José Urias Fortes, 245, ap. 32, Bairro São Manoel CEP 15091-220 - São José do Rio Preto (SP), Brasil

Tel.: (11) 97654-5110

E-mail: carolinavascsantanna@hotmail.com

Informações sobre os autores CVS, FGK, VBF e MRC - Alunos do $6^{\circ}$ período de Medicina, Faculdade de Medicina de São José do Rio Preto (FAMERP). AML - Graduada em Medicina, Universidade do Estado do Pará, Residente em Cirurgia Geral e Cirurgia Vascular e Angiologia, Faculdade de Medicina de São José do Rio Preto (FAMERP).

SROR - Graduada em Medicina e Mestre em Ciências da Saúde, Faculdade de Medicina de São José do Rio Preto (FAMERP);

Professora Adjunta II-M, Serviço de Cirurgia Vascular e Angiologia, Departamento de Cardiologia e Cirurgia Cardiovascular da FAMERP;

Titular da Sociedade Brasileira de Angiologia e Cirurgia Vascular

(SBACV), Especialista em Angiologia e Cirurgia Vascular da SBACV;

Médica, Hospital de Base de São José do Rio Preto e do Hospital Austa de São José do Rio Preto. ARM - Graduado em Medicina, Faculdade de Medicina de São José do Rio Preto (FAMERP); Médico, Hospital de Base de São José do Rio Preto; Titular da Sociedade Brasileira de Angiologia e Cirurgia Vascular

(SBACV). Especialista em Angiologia e Cirurgia Vascular da SBACV; Especialista em Angiorradiologia e Cirurgia Endovascular:; VA - Aluna do $5^{\circ}$ período de Medicina, Faculdade de Medicina de São José do Rio Preto (FAMERP).

Contribuição dos autores Concepção e desenho do estudo: CVS, FGK, AML, SROR, ARM, VBF Análise e interpretação dos dados: CVS, FGK, AML, VBF, MRC Coleta de dados: CVS, FGK, AML, VA, MRC Redação do artigo: CVS, FGK, AML, VA, SROR Revisão crítica do texto: AML, SROR, ARM Aprovação final do artigo*: CVS, FGK, AML, SROR, ARM, VA, VBF, $M R C$ Análise estatística: N/A. Responsabilidade geral pelo estudo: CVS, SROR

*Todos os autores leram e aprovaram a versão final submetida ao J Vasc Bras. 